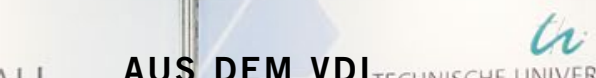

\title{
FISITA World Automotive Congress in Süd-Korea
}
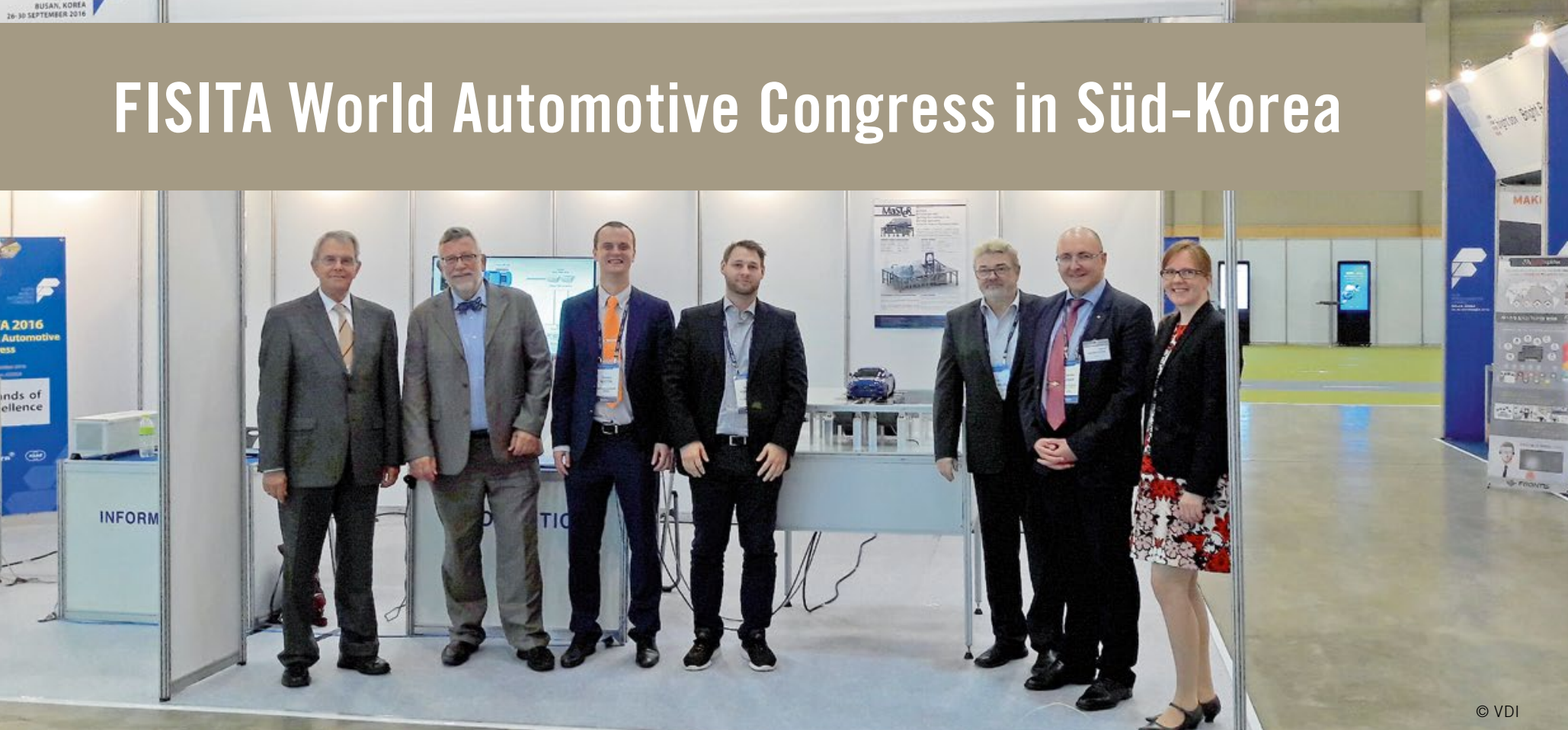

VDI besucht den Stand der TU IImenau, v.I.n.r.: Detlef Frank (VDI-FVT-Beirat), Dr. Ludwig Vollrath (FISITA Vice President Europe), Dzmitry Savitski und Viktor Schreiber (beide Wissenschaftliche Mitarbeiter Fachgebiet Kraftfahrzeugtechnik), Prof. Klaus Augsburg (Leiter Fachgebiet Kraftfahrzeugtechnik), Prof. Valentin Ivanov (Wissenschaftlicher Mitarbeiter Fachgebiet Kraftfahrzeugtechnik), Dr. Daniela Hein (Geschäftsführerin VDI-GPP und FISITA Vice President Education)

\section{Vom 26.-30. September 2016 fand in Busan, Süd-Korea, der 36. FISITA World Automotive Congress} statt. Die Technische Universität IImenau demonstrierte im Rahmen der Fachausstellung einen neuartigen methodischen Ansatz zur Erforschung von Fahrzeugsystemen.

\section{SCHNITTSTELLEN}

Die Komplexität fahrzeugtechnischer Systeme erfordert interdisziplinär vernetzte Ansätze. Die TU Ilmenau wendet sich in einer ihrer strategischen Forschungslinien der Vernetzung von experimentellen Plattformen und der Kosimulation analytischer Entwicklungstools in Echtzeit zu. Diese Vernetzung bildet die Grundlage zur parallelen Entwicklung von Fahrzeugsystemen auch über größere räumliche Distanzen. Eine Voraussetzung dafür ist die Definition von echtzeitfähigen, einheitlichen Schnittstellen. Diese Aufgabe ist Teil der aktuellen Forschungsarbeiten im Fachgebiet Kraftfahrzeugtechnik der Technischen Universität Ilmenau, die in enger Kooperation mit der Industrie (AVL, dSpace, Bosch, etc.) in europäischen Projekten wie ACOSAR und EVE bearbeitet werden.

Die Erprobung der Vernetzungstechnologien wird künftig über einen skalierten Modellansatz erfolgen, bei dem die Prüfstände im Modellmaßstab realisiert sind.

\section{ISLANDS OF EXCELLENCE}

Mit diesem Ansatz haben Wissenschaftler, Promovenden und Studierende der TU Ilmenau am Wettbewerb Islands of Excellence des FISITA World Automotive Congress teilgenommen. Die FISITA ist der internationale Dachverband aller Automobilingenieurverbände. Universitäten und Forschungseinrichtungen können sich alle zwei Jahre darum bewerben, eine sogenannte Island of Excellence zu gestalten. Eine Expertenjury bewertet die Konzepte und wählt aus den weltweiten Einreichungen die besten aus. In diesem Jahr erhielten neben der TU Ilmenau die TU Delft sowie die Kookmin University und die Seoul National University Gelegenheit, im Rahmen der begleitenden Fachausstellung des Kongresses ihre zukunftsweisenden, visionären Projekte einem internationalen Fachpublikum vorzustellen.

\section{DEMONSTRATOR DER TU ILMENAU}

Zu sehen war ein Demonstrator, der die Vernetzung am Beispiel eines im Maßstab 1:10 skalierten Vier-Rollen-Prüfstands und Bremsenprüfstands repräsentiert. Diesem ist eine echtzeitfähige Gesamtfahrzeugsimulation übergeordnet, die einzelne Fahrzeugteilsysteme, Fahrzeugparameter, die Fahrumgebung und ein Testszenario abbildet. Durch die Substitution der virtuellen Teilsysteme im Simulationsmodell mit realen Komponenten auf Prüfständen wird eine Prüfumgebung geschaffen, die physikalische Effekte präziser berücksichtigt. Dabei ist es sehr wichtig, dass alle Komponenten in Echtzeit arbeiten.

Weitere Informationen unter: www.fisita.com

www.tu-ilmenau.de/kft/

www.vdi.de/fvt 